\title{
Microscopic Picture of Calcium-Assisted Lipid Demixing and Membrane Remodeling Using Multiscale Simulations
}

\author{
Abhilash Sahoo ${ }^{\dagger}$ and Silvina Matysiak*,†, $\ddagger$ \\ †Biophysics Program, Institute of Physical Science and Technology, University of \\ Maryland, College Park, MD \\ $\ddagger$ Fischell Department of Bioengineering, University of Maryland, College Park, MD \\ E-mail: matysiak@umd.edu
}




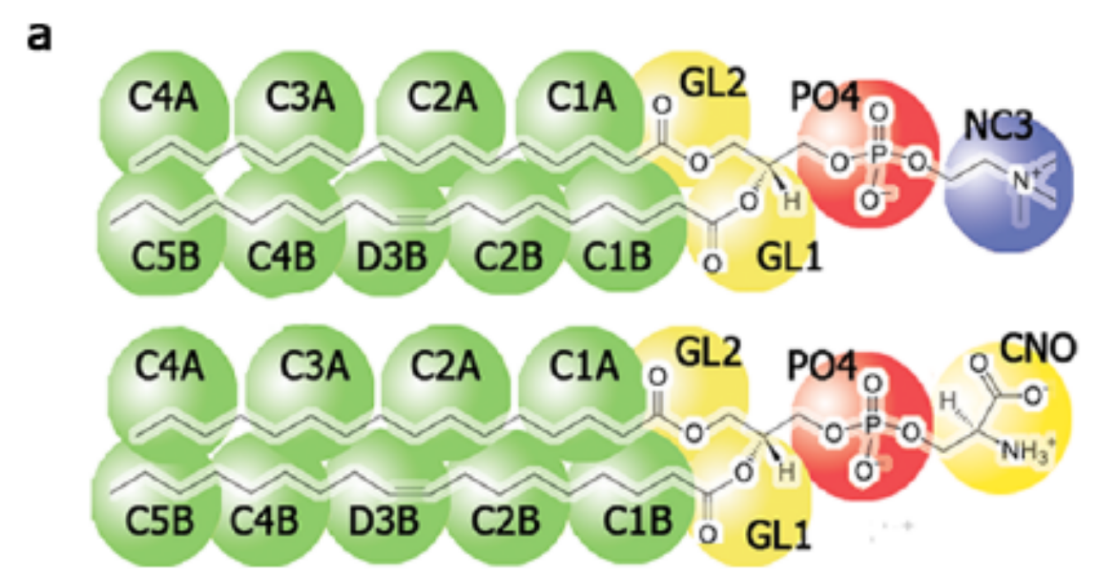

b

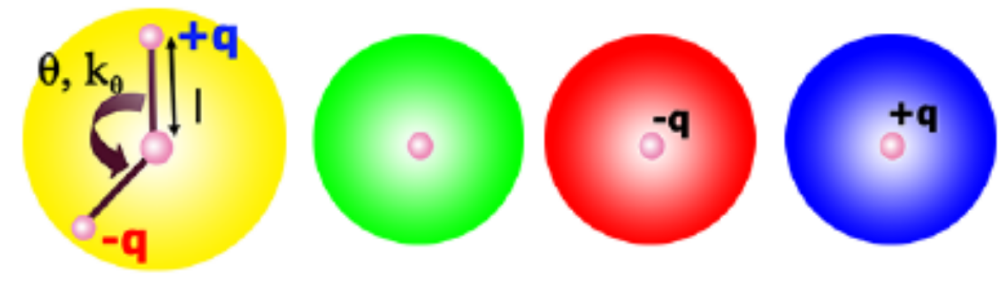

Figure S1: (a) Mapping scheme for POPC and POPS coarse-grained lipids; colors: green, hydrophobic bead; yellow, polarizable beads ; red, negatively charged bead; blue, positively charged bead. (b) Bead types: yellow represents polarizable beads (example : CNO, GL1, GL2), van der Waals radius of the bead encloses positively $(+q / P)$ and negatively $(-q / M)$ charged dummy particles (example:, CNP and CNM, G1P and G1M, G2P and G2M respectively). Blue bead represents a positively charged bead, red bead represents negatively charged bead, and green represents hydrophobic bead. Image reproduced from Ganesan et.al. ${ }^{1}$ with permission from the PCCP Owner Societies. 


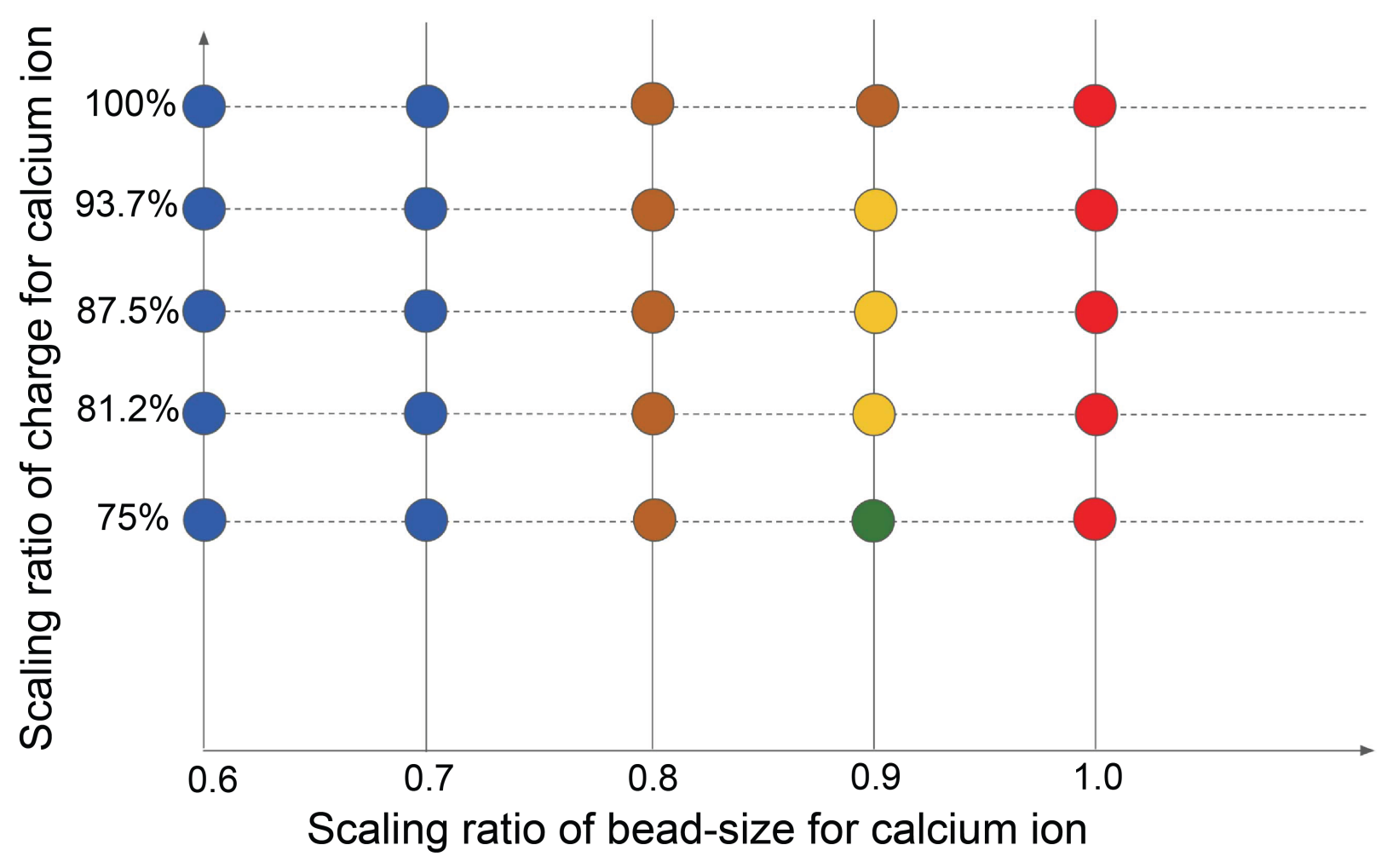

Figure S2: Schematic description of simulations for parametrization of calcium ion. Each colored circle represents a 500 ns simulation. Red circles represent simulations that could not capture appropriate partitioning of calcium ions into membrane. Dark-orange and yellow circles represent simulations that captured an effective median lipid coordination of 3, contrary to previous reports. The difference between simulations represented by dark-orange and yellow circles is the absence of lipid-coordination diversity (coordination number of 1, 2, 3 and 4) as observed in previous publications. The blue circles denote simulations which could not reproduce this diversity of lipid-coordination. The simulation represented by the green circle captured the median lipid-coordination of 2 and similar diversity in lipid-coordination as in previous reports and backmapped simulations. 


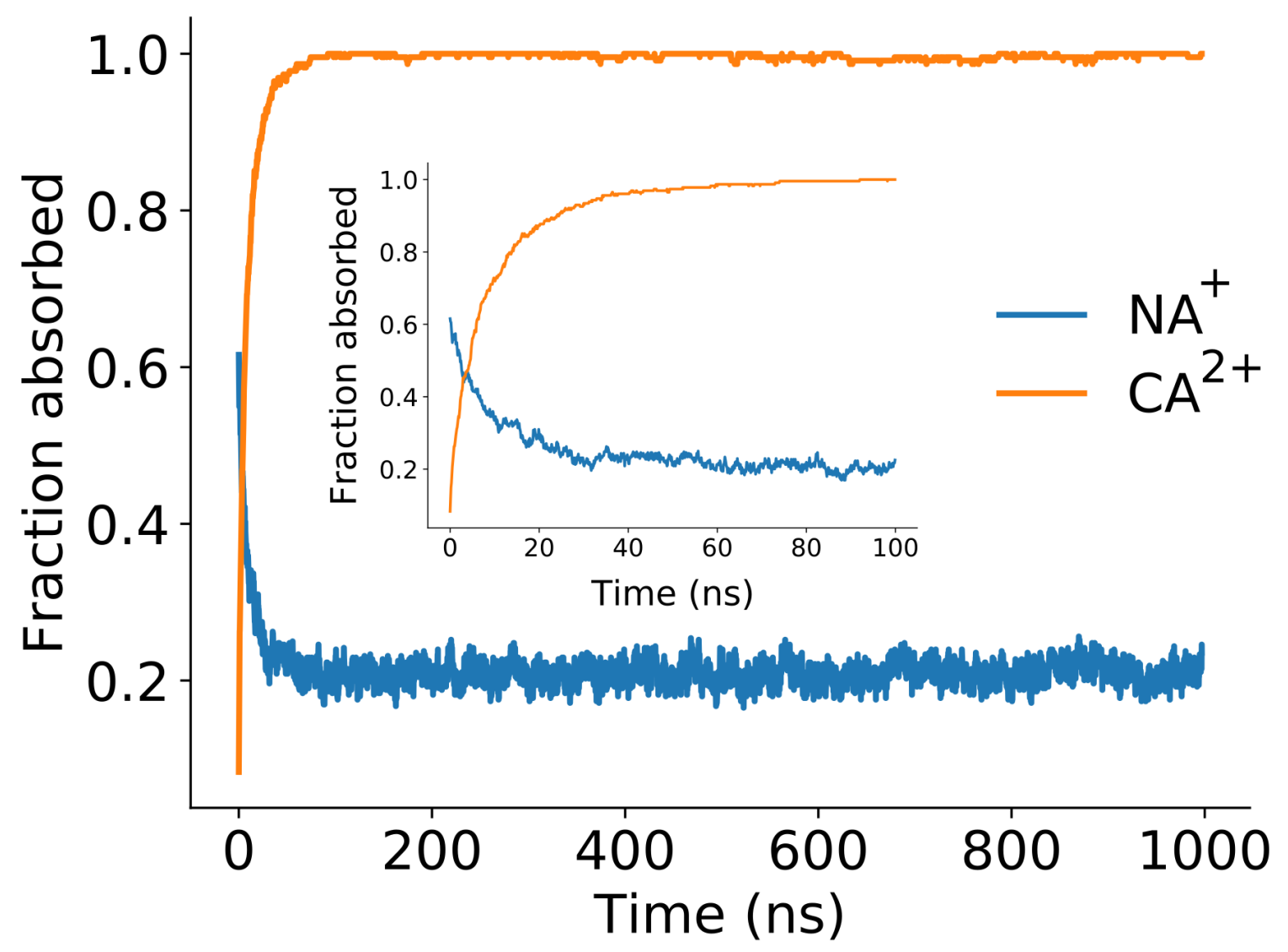

Figure S3: Fraction of absorbed calcium and sodium ions over time. inset - Fraction of absorbed ions over the initial $100 \mathrm{~ns}$. 
a

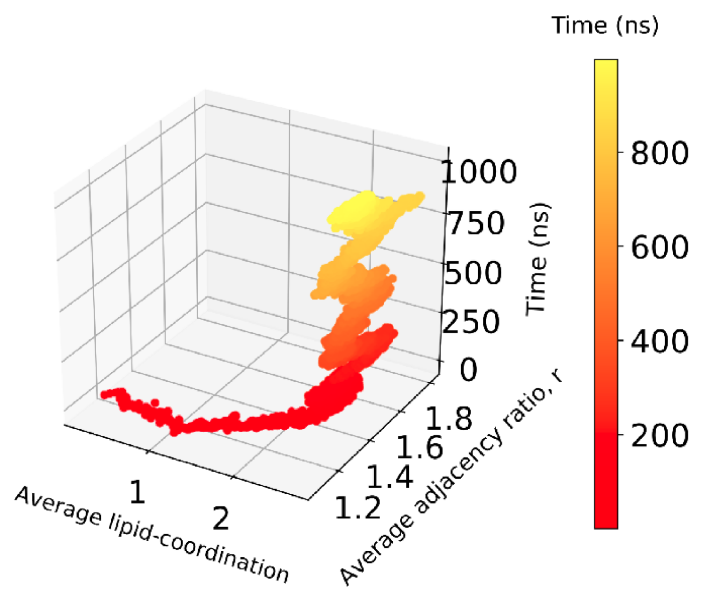

C

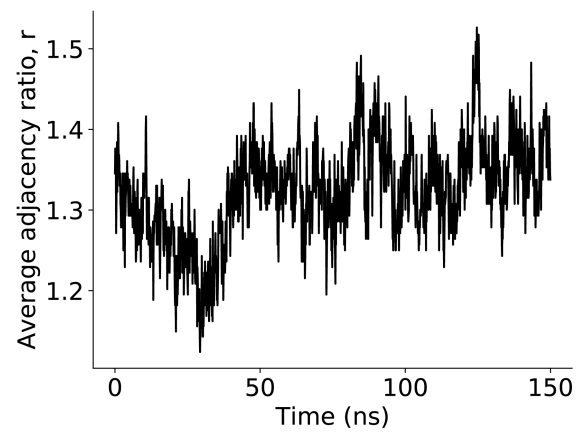

b

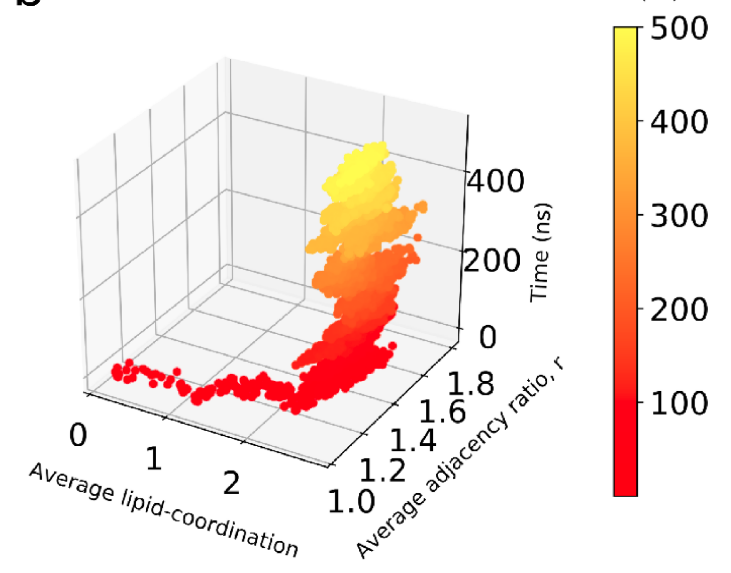

d

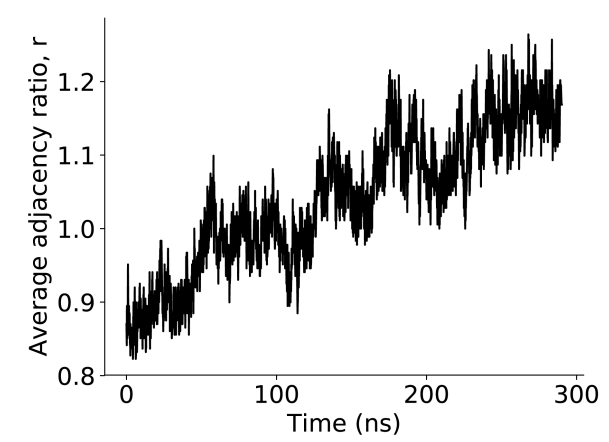

Figure S4: Co-dependence plot between average-lipid-coordination and average-adjacencyratio (r) over time of 968 (a) and 240 (b) lipid system. c) Variation of average adjacency ratio, $r$ over time for backmapped simulation. d) Variation of average adjacency ratio, $r$ over time for RI simulation. Average adjacency ratio, $r$ increases over time for RI simulation suggesting that head-group equillibration has not been reached. 
Table S1: Number of water molecules within the first interaction peak of lipids - POPC and POPS

\begin{tabular}{lccc}
\hline & Backmapped-atomistic & Randomly-initiated (RI) & No-calcium \\
\hline POPC & $8.244 \pm 0.283$ & $8.232 \pm 0.252$ & $8.325 \pm 0.302$ \\
POPS & $5.667 \pm 0.192$ & $5.471 \pm 0.166$ & $6.015 \pm 0.190$ \\
\hline
\end{tabular}


a

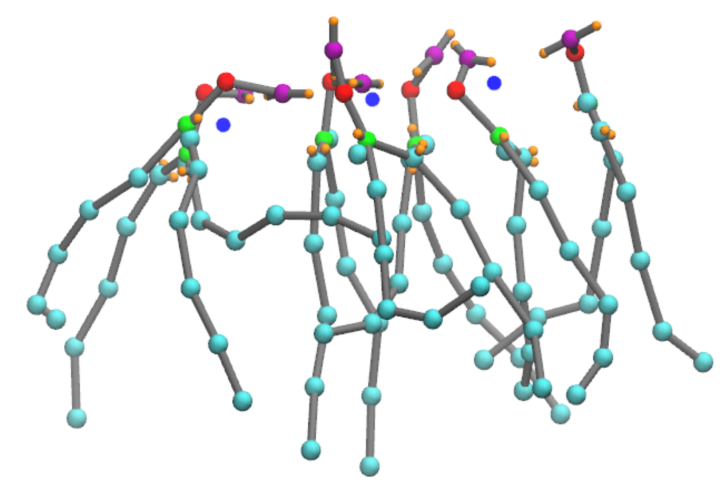

b

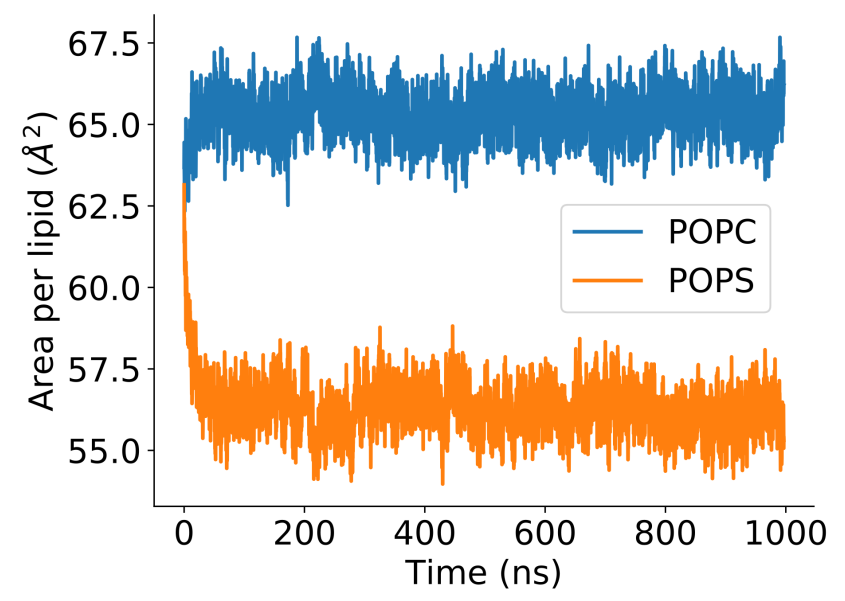

C

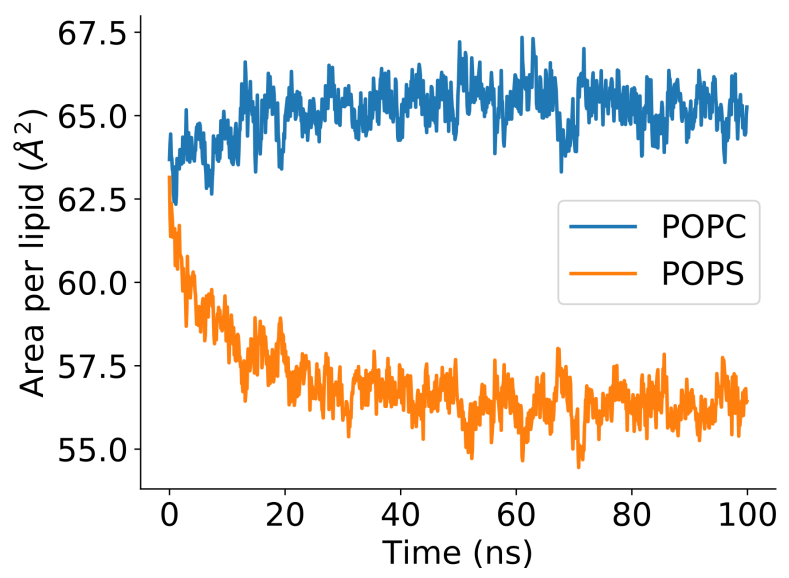

Figure S5: a) Truncated-cone like structure of POPS lipids due to interaction with calcium (in blue). Color code: Serine - Purple; Charged dipole beads - Orange; Phosphate - Red; Glycerol-ester beads - Green; Acyl tails - Cyan b) Variation of area-per-lipid with time. c) Variation of area-per-lipid with time over the first $100 \mathrm{~ns}$. 


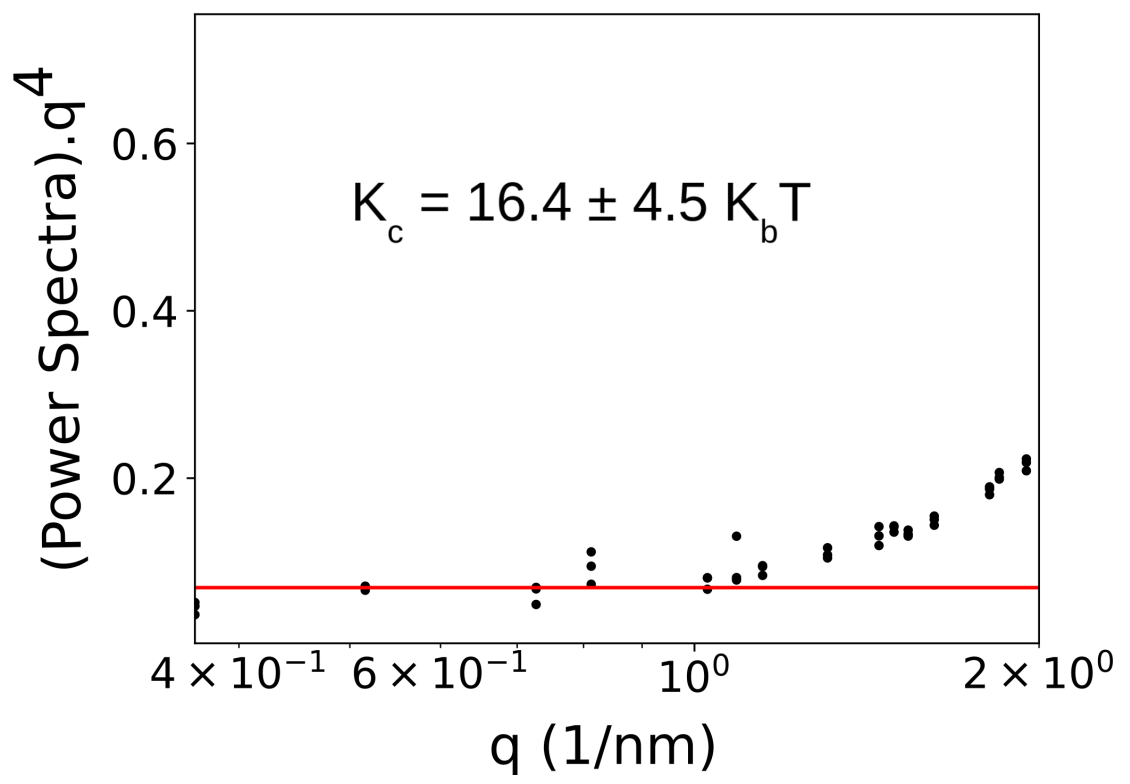

Figure S6: Asymptotic fit to long wavelength power spectra of height field. Bending modulus can be derived from Helfrich-Canham (HC) theory - $\left\langle|h(q)|^{2}\right\rangle=\frac{K_{b} T}{K_{c} q^{4}}$ 


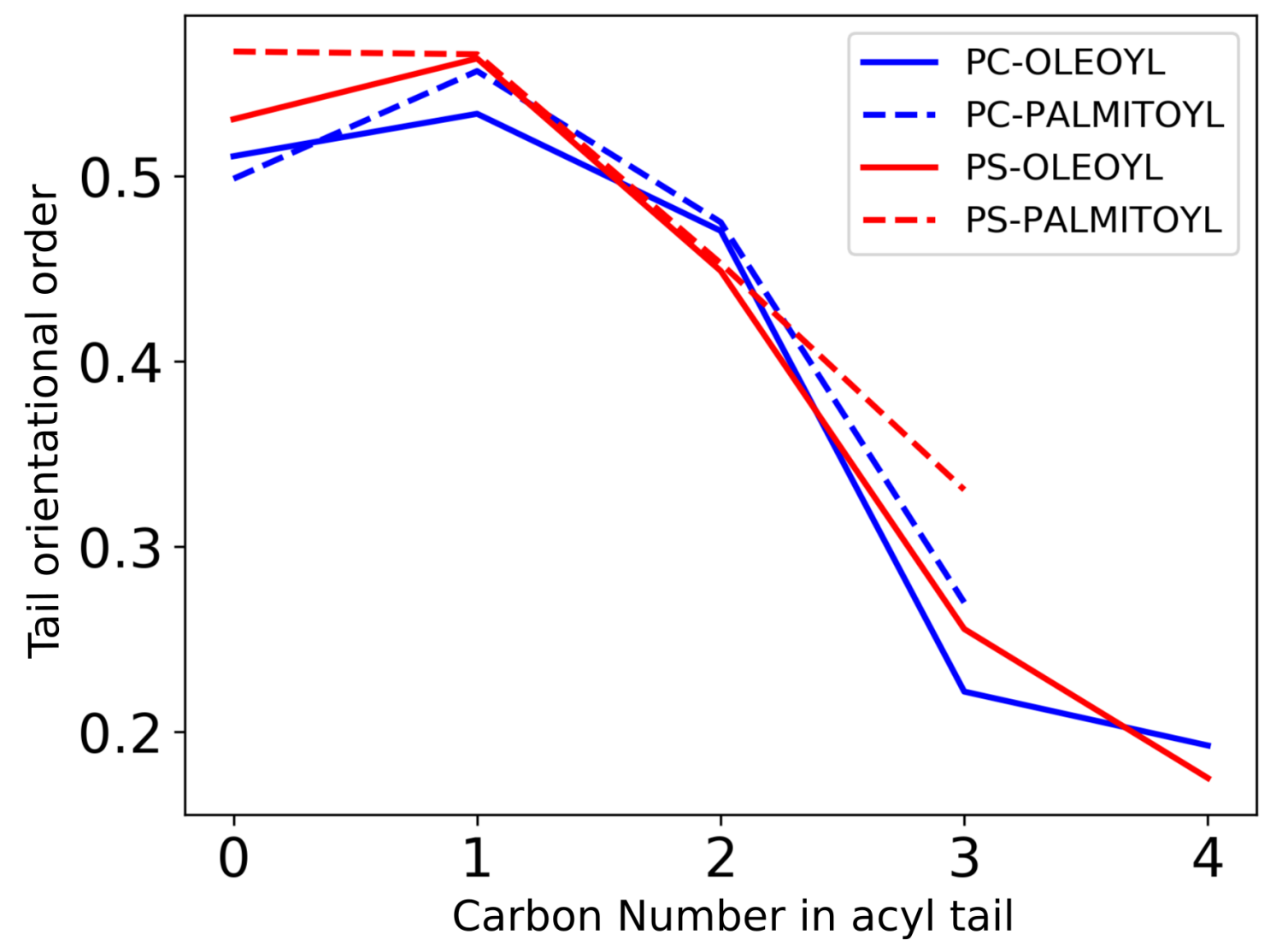

Figure S7: Tail orientational order parameter $-S_{z}=\left\langle\frac{1}{2}\left(3 \cos ^{2} \theta-1\right)\right\rangle$, where $\theta$ is the tilt of the lipid tail with bilayer normal.

\section{References}

(1) Ganesan, S. J.; Xu, H.; Matysiak, S. Effect of lipid head group interactions on membrane properties and membrane-induced cationic $\beta$-hairpin folding. Phys. Chem. Chem. Phys. 2016, 18, 17836-17850. 